\section{ACMG recommendations on incidental findings are flawed scientifically and ethically}

To the Editor: The "ACMG Recommendations for Reporting of Incidental Findings in Clinical Exome and Genome Sequencing" maintain that patients who consent to have their own or their child's whole genome or exome sequenced should be informed of certain incidental findings $(n=57)$ "without reference to patient preferences." A recent supporting paper noted, "In the event of malpractice litigation, the recommendations may be introduced as evidence of the standard of care." ${ }^{32}$ This is premature because the recommendations are flawed scientifically as well as ethically. They are based on the beliefs that the presence of these incidental findings indicates that the patient or his/her relatives will suffer future harm and that interventions are available to reduce or prevent harm. The evidence to support these beliefs is insufficient to constitute reporting them as "the standard of care."

The 57 incidental findings listed in the American College of Medical Genetics and Genomics (ACMG) recommendations were derived from the opinions of 16 clinical geneticists and/or molecular laboratory directors ${ }^{3}$ and "supplemented by a provisional list of genes." The 16 raters "were told to assume that the sequencing was perfectly accurate ... even though this degree of accuracy is not available through current WES/WGS [whole-exome sequencing/whole-genome sequencing] technologies." ${ }^{\text {"3 }}$ Each finding is a sequence variation that has been "previously reported and is a recognized cause of the disorder" or is a previously unreported sequence "of the type expected to cause the disorder." Of the 57 findings, 46 were rated in the study by Green et al. ${ }^{3}$ Two more (Ehlers-Danlos; catecholaminergic polymorphic ventricular tachycardia) were rated as "Bin 1" (reportable) by Berg et $\mathrm{al}^{4}{ }^{4}$ (I could not find the source for the nine remaining findings for which reporting is mandatory. They are WT1related Wilms' tumor (one gene), neurofibromatosis type 2 (one gene), arrhythmogenic right-ventricular cardiomyopathy (five genes), and malignant hyperthermia susceptibility (two genes).)

In only 14 of the 46 incidental findings rated in the study by Green et al. ${ }^{3}$ did all raters agree that the finding in both adults and children should be reported; in 12 others, $100 \%$ consensus was attained for reporting their presence in adults only. Agreement to report was lower when raters were asked to rate previously unknown truncating and missense variants. When experts disagree, how can the reporting of these findings be mandatory?
The previously reported sequence variations have been found in families in which at least one individual expresses the disease phenotype. But as incidental findings, the sequence variations are likely to be found in individuals without a family history. We do not know if any of the reportable variations will have as high penetrance in such families as in those in whom the disease phenotype is present; there could be modifying genes or gene copies that reduce penetrance. The same is even more likely for previously unreported variations for which no genotype-phenotype relation has been established. Somewhat cryptically, Green et al. ${ }^{1}$ acknowledge these problems: "As additional evidence accrues on the penetrance of these variants among persons without symptoms or family history, these recommendations will be expected to evolve."' I interpret this to mean that the incidental presence of some of these variations could be false positives; individuals will then have been compelled to receive erroneous information. With such uncertainty, it seems wise to (i) treat the reporting of "pathogenic" sequence variations as research; (ii) give patients who consent to whole-genome sequencing/ whole-exome sequencing the opportunity to consent to being informed, or not, of incidental findings, having been told of the possible benefits and the unknown probability of false positives (and false negatives); and (iii) have patients who consent to being informed of incidental findings enrolled in a data registry for the purpose of collecting evidence of a genotype-phenotype relationship and whether the expected phenotype can be prevented or ameliorated by intervention. The ACMG recommendations do not include reporting to a data registry, although the authors acknowledged "a great need to develop a central repository of genotypic and phenotypic data."

What is the rush to mandate informing patients, or their providers, of incidental findings before "the benefits, harms and costs that may result" have been established? Green et al. ${ }^{1}$ have either not heard of the precautionary principle or have chosen to disregard it. That is, perhaps, the most unethical aspect of the ACMG recommendations.

There are additional uncertainties. Lam et al. ${ }^{6}$ found that the two most frequently used sequencing platforms are not in perfect agreement in detecting variations. For example, "We detected 444,678 SNVs (single-nucleotide variants) by only one platform or the other but not both." Although confirmatory techniques, such as Sanger sequencing, can reduce or eliminate analytical false positives, they cannot eliminate analytical false negatives. The ACMG should put the horse before the cart and work toward improving the reliability of sequencing in clinical laboratories, as well as toward laboratory quality control and ensurance of appropriate genetic counseling, before making pronouncements on incidental findings in clinical practice.

Green et al. ${ }^{1}$ invert the importance of returning incidental findings in research as compared with practice. "There is," they say, "an active debate about the return of incidental findings 
in genomic research," but "these recommendations [are] for the situation in which a clinician orders exome or genome sequencing for a specific clinical indication. In this circumstance, a laboratory report will be returned to that clinician" (my emphasis). It is hard to understand why there should be less debate about returning incidental findings in clinical practice than in research, given the uncertainties discussed above. Clinical practice should require a more rigorous debate, and it should be performed before recommendations that "may be introduced as evidence of the standard of care" 2 are promulgated. Truncating debate will have as serious consequences for society as truncating sequences may have for individuals. The ACMG should rescind these recommendations and proceed more cautiously.

\section{DISCLOSURE}

The author declares no conflict of interest.

\section{Neil A. Holtzman, $M D, M P H^{1}$}

${ }^{1}$ Department of Pediatrics, Institute of Genetic Medicine, The Johns Hopkins University School of Medicine, Baltimore, Maryland, USA. Correspondence: Neil A. Holtzman (nholtzma@jhsph.edu)

\section{REFERENCES}

1. Green RC, Berg, JS, Grody WW, et al. ACMG recommendations for reporting of incidental findings in clinical exome and genome sequencing. Genet Med 2013;15:565-574.

2. McGuire AL, Joffe $S$, Koenig BA, et al. Ethics and genomic incidental findings. Science 2013;340:1047-1048.

3. Green RC, Berg JS, Berry GT, et al. Exploring concordance and discordance for return of incidental findings from clinical sequencing. Genet Med 2012;14: 405-410.

4. Berg JS, Adams M, Nassar N, et al. An informatics approach to analyzing the incidentalome. Genet Med 2013;15:36-44.

5. American College of Medical Genetics and Genomics. Incidental findings in clinical genomics: a clarification. 2 May 2013. http://www.acmg.net/docs/ Incidental_Findings_in_Clinical_Genomics_A_Clarification.pdf.

6. Lam HY, Clark MJ, Chen R, et al. Performance comparison of whole-genome sequencing platforms. Nat Biotechno/ 2012;30:78-82.

doi:10.1038/gim.2013.96

\section{Paternalism and the ACMG recommendations on genomic incidental findings: patients seen but not heard}

To the Editor: Incidental findings that may arise in wholeexome or whole-genome sequencing pose significant challenges for clinical care. The American College of Medical Genetics and Genomics' (ACMG's) recent article, "ACMG Recommendations for Reporting of Incidental Findings in Clinical Exome and Genome Sequencing," establishes routine analysis of pathogenic variants of a list of disease-associated genetic loci as a standard of practice when clinical whole-genome sequencing is done for any reason except prenatal testing. ${ }^{1}$ The findings are to be reported to the ordering physician for disclosure to the patient (or if a child, to his/her parents). This recommendation reverses current practice that supports the patient's right to choose not to be informed of incidental genetic information.

The year-long consensus process used to develop the recommendations involved extensive discussions among an ACMG Working Group, review by an independent group of experts, and approval by the ACMG Board of Directors. The individuals involved are all well-qualified representatives of the medical, clinical laboratory, and genetic counseling communities. Conspicuously absent from the ACMG process, however, are the voices of patients and families who might need whole-exome or -genome sequencing to diagnose a serious medical condition. This is a concern because a key ethical principle in devising effective and practical clinical recommendations is that they are fair, which means that all individuals affected by the recommendations should contribute to their development.

Ethical concerns underpin clinical genetics but are compressed in these recommendations in which the professionals' fiduciary duty trumps patient autonomy: "Clinicians and laboratory personnel have a fiduciary duty to prevent harm by warning patients and their families about certain incidental findings and that this principle supersedes concerns about autonomy, just as it does elsewhere in medical practice." The physician-patient relationship is based on trust and responsibility, but it is not an ethical principle, nor is it a fixed concept. The traditionally paternalistic model of medicine, underpinned by values and assumptions about passive patient and authoritative physician roles, is increasingly criticized by patients, advocacy groups, health policy makers, and many physicians. Notions of trust and the fiduciary relationship are shifting as medical practice engages patients, offers transparency of information, and encourages more patient responsibility for the choices made.

We also question the ACMG Working Group's contention that routine disclosure of the results of a set of genetic analyses that is actively sought in every case is no different from reporting the incidental discovery of an unexpected disease manifestation in other clinical contexts. When physicians perform a complete medical history and physical examination or carefully review the entire field revealed by an imaging study, they are looking for signs of disease that is already present in a particular patient. By contrast, the ACMG recommendations require looking for mutations that predict diseases that have not yet occurred in each patient who is tested.

The ACMG Working Group acknowledges that their recommendations are not evidence based: adequate evidence regarding the best way to return genomic incidental findings does not yet exist. Nevertheless, the evidence that is available-some of which was neglected in the recommendations-should be considered before endorsing disclosure to patients regardless of their preferences. Many people choose not to learn about 